\title{
Solubility of cefoxitin acid in different solvent systems
}

Fuhong Yuan ${ }^{\mathrm{a}}$, Yongli Wang ${ }^{\mathrm{a}, \mathrm{b}^{*}}$, Liping Xiao ${ }^{\mathrm{a}}$, Qiaoyin Huang ${ }^{\mathrm{a}}$, Jinchao Xu ${ }^{\mathrm{a}}$, Chen Jiang ${ }^{\mathrm{a}}$, Hongxun $\mathrm{Hao}^{\mathrm{a}, \mathrm{b}^{*}}$

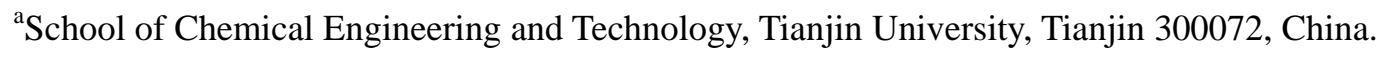

${ }^{\mathrm{b}}$ The Co-Innovation Center of Chemistry and Chemical Engineering of Tianjin, Tianjin 300072,

China.

*Corresponding author. Tel.: +86-22-27405754; Fax: +86-22-27374971.

E-mail address: hongxunhao@tju.edu.cn, yliwang@tju.edu.cn.

ABSTRACT: Cefoxitin acid is one kind of important pharmaceutical intermediate.

Its solubility is crucial for designing and optimizing the crystallization processes. In this work, the solubility of cefoxitin acid in organic solvents (methanol, acetonitrile, ethanol, isopropanol, n-propanol and ethyl acetate), water and water-methanol mixtures was measured spectrophotometrically using a shake-flask method within the temperature range $278.15 \mathrm{~K}$ to $303.15 \mathrm{~K}$. PXRD data and the Karl Fischer method were used to verify the crystal form stability of cefoxitin acid in the solubility measuring process. The melting points, the enthalpy and entropy of fusion were estimated. Results showed that the solubility of cefoxitin acid increases with the increasing temperature in all tested solvents in this work, and the solubility of 
cefoxitin acid increases with the increasing methanol concentration in water-methanol mixtures. The experimental solubility values were well correlated using the modified Apelblat equation, NRTL model and CNIBS/R-K model. An equation proposed by Williamson was adopted to calculate the molar enthalpy during the dissolution process.

Keywords: cefoxitin acid; anhydrate; hydrate; solubility; dissolution enthalpy

\section{Introduction}

Cefoxitin acid ((6R)-3-carbamoyloxymethyl-7c-methoxy-8-oxo-7t-(2-thiophen-2 -yl-acetylamino)-(6rH-5-thia-1-aza-bicyclo[4.2.0]oct-2-ene-2-carboxylic acid, CAS No. 35607-66-0) (figure1) is a semi-synthetic cephamycin and possesses important biochemical characteristics that are not found in other $\beta$-lactam antibiotics due to its $7 c$-methoxyl group [1,2]. It is also an important intermediate for the production of cefoxitin sodium in the pharmaceutical industry. Since the quality of cefoxitin acid is largely affected by the purification and crystallization process, it is crucial to develop and design an optimized crystallization process for cefoxitin. Therefore, a knowledge of the solubility which is essential for crystallization process design needs to be determined $[3,4]$. However, solubility of cefoxitin acid in various solvents at different temperatures has not been reported until now.

In this work, the solubility of cefoxitin acid in methanol, acetonitrile, ethanol, 
isopropanol, n-propanol, ethyl acetate, water and water-methanol mixtures was determined from $278.15 \mathrm{~K}$ to $308.15 \mathrm{~K}$ by ultraviolet spectrophotometry (UV) under atmospheric pressure.

\section{Experimental}

\subsection{Materials}

Cefoxitin acid monohydrate (0.995 mass fraction purity) was supplied by Hebei Huamin Pharmaceutical Co., Ltd. of China. The water content of the purchased cefoxitin acid monohydrate was determined to be $(4.12 \pm 0.2)$ mass $\%$ with a V20 Volumetric Karl Fischer Titrator from Mettler Toledo. Cefoxitin acid anhydrate was prepared by anti-solvent crystallization in water-methanol mixtures, using the aforementioned cefoxitin acid hydrate as raw material. Cefoxitin acid hydrate $(2.4 \mathrm{~g})$ was completely dissolved with $50 \mathrm{~mL}$ methanol in a glass jacketed crystallizer with agitation at $300 \mathrm{rpm}$ and temperature of $283.15 \mathrm{~K}$. Water $(60 \mathrm{~mL})$ was added to the solution with dosing rate of $1 \mathrm{~mL} \cdot \mathrm{min}^{-1}$. The product was filtered and washed with water for several times, and finally dried at $303.15 \mathrm{~K}$ for $24 \mathrm{~h}$ in a vacuum oven. The identification of the product obtained was confirmed to be anhydrous cefoxitin acid by PXRD analysis. The mass fraction purity of cefoxitin acid anhydrate is above 0.998 which was determined by high performance liquid chromatography (HPLC) (Agilent 1200 Series system, U.S.A.). The water content of cefoxitin acid anhydrate was determined to be $(0.20 \pm 0.2)$ mass $\%$. The organic solvents (methanol, acetonitrile, ethanol, isopropanol, 1-propanol and ethyl acetate) used in this study are 
analytical reagent. Water for all experiments was purchased from Nankai University, China. The conductivity of the used water was $0.055 \mu \mathrm{S} \cdot \mathrm{cm}^{-1}$. All the solvents were used without further purification or prior treatment. More details of the materials are listed in table 1.

2.2. Characterization of cefoxitin acid anhydrate and its hydrate

Cefoxitin acid anhydrate and its hydrate were characterized by the powder X-ray diffraction (PXRD), Tabletop microscopy, Thermo Gravimetric Analyzer and Differential Scanning Calorimetry Synchronous Analyzer (TGA/DSC), and Raman spectroscopy. The PXRD was obtained by using $\mathrm{Cu} \mathrm{K} \alpha$ radiation $\left(1.54 \times 10^{-8} \mathrm{~cm}\right)$ on a Rigaku D/max-2500 (Rigaku, Japan). The analyses were carried out over a 2-theta range of $(2-40)^{\circ}$ at a step size of $0.02^{\circ}$, a voltage of $40 \mathrm{kV}$, and an electric current of $100 \mathrm{~mA}$. The crystal habits were observed using a Tabletop Microscope (TM3000, HITACHI, Japan). The thermal analysis experiment was carried out by using a TGA/DSC simultaneous thermal analyser (Model TGA/DSC 1, Mettler-Toledo, Switzerland), which was carried out with a heating rate of $10 \mathrm{~K} \cdot \mathrm{min}^{-1}$ under the protection of nitrogen. The Raman spectra were collected with a Kaiser RamanRXN2 Hybrid System from Kaiser Optical System, Inc. The $\mathrm{P}^{\mathrm{h}} \mathrm{AT}$ probe was used to ex situ collect the Raman spectra of cefoxitin acid anhydrate and its hydrate.

\subsection{Solubility measurements}

The solubility of cefoxitin acid was measured by a shake-flask method. The measurements were carried out in a METTLER TOLEDO EasyMax ${ }^{\mathrm{TM}} 102$ 
Workstation (hereinafter referred to as EasyMax ${ }^{\mathrm{TM}}$ ). The EasyMax ${ }^{\mathrm{TM}}$ can be used to perform multiple solubility experiments since it has 6 heavy walled borosilicate glass tubes (volume of $25 \mathrm{~mL}$ ). A magnetic stir bar was used to mix the solution. A type $T$ thermocouple was used for monitoring the temperature inside the glass tubes with a resolution of $0.05 \mathrm{~K}$. For the methanol-water mixtures $(0.1013,0.2011,0.2933$, $0.4020,0.5003,0.5993$ mole fraction of methanol, $x 02$ ), prior to the solubility experiments, the solvent mixtures were prepared using an analytical balance with a resolution of $0.0001 \mathrm{~g}$. During the solubility experiments, $10 \mathrm{~mL}$ of the solvents (pure solvents or any of the aqueous mixtures) and excess solute (1 g, cefoxitin acid anhydrate or its hydrate) were poured into the corresponding glass tube of the EasyMax $^{\mathrm{TM}}$. Once the tube was carefully capped to avoid evaporation, the solute-solvent mixture was continuously agitated at $300 \mathrm{rpm}$ at constant temperature. Time of agitation was preliminarily determined by measuring the concentration of the solute at different time intervals (from $4 \mathrm{~h}$ to $8 \mathrm{~h}$ ). Eight hours were proven to be enough to reach the solid-liquid equilibrium. After $8 \mathrm{~h}$ agitation, the saturated solution was kept static for $1 \mathrm{~h}$ at the same temperature, allowing suspended solids to settle down. Then, about $1 \mathrm{~mL}$ sample was taken from the supernatant solution to a volumetric flask with a preheated micropipette and quickly weighted using the analytical balance. The withdrawn cefoxitin acid organic saturated solutions were diluted with corresponding organic solvent while the cefoxitin acid methanol aqueous saturated solutions were diluted with phosphate buffer to an appropriate concentration for UV analysis. Absorbance of standard solutions and samples were determined at 
$262 \mathrm{~nm}$ by an U-3010 UV-VIS Spectrophotometer (Hitachi High-technologies Co., Tokyo, Japan). The true absorbance at $262 \mathrm{~nm}$ was obtained by applying a suitable baseline correction and solvent deduction $[5,6]$.

The mole fraction solubility of cefoxitin acid in organic solvent was obtained from Eq.(1).

$x_{1}=\frac{m_{1} / M_{1}}{m_{1} / M_{1}+m_{2} / M_{2}}$

where $x_{1}$ is the mole fraction solubility of cefoxitin acid, $m_{1}$ and $M_{1}$ are the mass and molar mass of cefoxitin acid, respectively; $m_{2}$ and $M_{2}$ are the mass and molar mass of the solvent, respectively.

The mole fraction solubility of cefoxitin acid in water and methanol-water mixtures was obtained from Eq.(2).

$x_{1}=\frac{m_{1} / M_{1}}{m_{1} / M_{1}+m_{2} / M_{2}+m_{3} / M_{3}}$

where $x_{1}$ is the mole fraction solubility of cefoxitin acid, $m_{1}, m_{2}$, and $m_{3}$ are the mass of cefoxitin acid, methanol and water, respectively. $M_{1}, M_{2}$ and $M_{3}$ are the molar mass of cefoxitin acid, methanol and water, respectively. The value of $m_{3}$ includes the initial water mass and additional water mass from the dissolved hydrate.

Each experiment was performed three times and the average value was used to calculate the solubility. The residual powder of solute were filtered, dried and then analysed by PXRD and Karl Fischer titration. 


\section{Models for solubility of cefoxitin acid}

\subsection{The modified Apelblat equation}

The experimental solubility values can be correlated as a function of temperature by the modified Apelblat equation, an extensively used semi-empirical model as following [7].

$$
\ln x_{1}=A+\frac{B}{T / K}+C \ln (T / K)
$$

where $x_{1}$ and $T$ represent the mole fraction of the solute and the temperature of the solution, respectively. $A, B, C$ are the parameters of the modified Apelblat equation, which can be obtained by using a non-linear regression method.

\subsection{NRTL model}

The local composition models are widely applied to calculate the fugacity coefficients of solute in the solid-liquid equilibrium. A general simplified equation, which can be deduced from literature, is described in Eq.(4) [8].

$$
\ln x_{1}=\frac{\Delta H_{\mathrm{m}}}{R}\left(\frac{1}{T_{\mathrm{m}}}-\frac{1}{T}\right)-\ln \gamma_{1}
$$

where $x_{1}$ and $T$ represent the mole fraction of the solute and the temperature of the solution, respectively; $R$ is the gas constant; $\Delta H_{\mathrm{m}}$ and $T_{\mathrm{m}}$ stand for the melting enthalpy and melting temperature, respectively; $\gamma_{1}$ represents the activity coefficient of cefoxitin acid in the solvent. The activity coefficient of the solute calculated by NRTL 
model in the pure solvents or methanol-water mixtures can be obtained from the following equation.

$$
\begin{aligned}
\ln \gamma_{\mathrm{i}}= & \frac{\left(G_{\mathrm{ji}} x_{\mathrm{j}}+G_{\mathrm{kj}} x_{\mathrm{k}}\right)\left(\tau_{\mathrm{ji}} G_{\mathrm{ji}} x_{\mathrm{j}}+\tau_{\mathrm{ki}} G_{\mathrm{ki}} x_{\mathrm{k}}\right)}{\left(x_{\mathrm{i}}+G_{\mathrm{ji}} x_{\mathrm{j}}+x_{\mathrm{k}} G_{\mathrm{ki}} x_{\mathrm{k}}\right)^{2}} \\
& +\frac{\tau_{\mathrm{ij}} G_{\mathrm{ij}} x_{\mathrm{j}}^{2}+G_{\mathrm{ij}} G_{\mathrm{kj}} x_{\mathrm{j}} x_{\mathrm{k}}\left(\tau_{\mathrm{ij}}-\tau_{\mathrm{kj}}\right)}{\left(x_{\mathrm{j}}+x_{\mathrm{i}} G_{\mathrm{ij}}+x_{\mathrm{k}} G_{\mathrm{kj}}\right)^{2}} \\
& +\frac{\tau_{\mathrm{ik}} G_{\mathrm{ij}} x_{\mathrm{j}}^{2}+G_{\mathrm{ij}} G_{\mathrm{kj}} x_{\mathrm{j}} x_{\mathrm{k}}\left(\tau_{\mathrm{ik}}-\tau_{\mathrm{jk}}\right)}{\left(x_{\mathrm{k}}+x_{\mathrm{i}} G_{\mathrm{ik}}+x_{\mathrm{j}} G_{\mathrm{jk}}\right)^{2}}
\end{aligned}
$$

where $x_{\mathrm{i}}, x_{\mathrm{j}}$ and $x_{\mathrm{k}}$ represent the mole fraction of the component $i, j$ and $k$ in the solution. $G_{\mathrm{ij}}, G_{\mathrm{ik}}, G_{\mathrm{ji}}, G_{\mathrm{jk}}, G_{\mathrm{ki}}, G_{\mathrm{kj}}, \tau_{\mathrm{ij}}, \tau_{\mathrm{ik}}, \tau_{\mathrm{ji}}, \tau_{\mathrm{jk}}, \tau_{\mathrm{ki}}$, and $\tau_{\mathrm{kj}}$ are parameters of this model.

$$
\begin{array}{ll}
G_{\mathrm{ij}}=\exp \left(-\alpha_{\mathrm{ij}} \tau_{\mathrm{ij}}\right) & \tau_{\mathrm{ij}}=\frac{\left(g_{\mathrm{ij}}-g_{\mathrm{jj}}\right)}{R T}=\frac{\Delta \mathrm{g}_{\mathrm{ij}}}{R T} \\
G_{\mathrm{ik}}=\exp \left(-\alpha_{\mathrm{ik}} \tau_{\mathrm{ik}}\right) & \tau_{\mathrm{ik}}=\frac{\left(g_{\mathrm{ik}}-g_{\mathrm{kk}}\right)}{R T}=\frac{\Delta \mathrm{g}_{\mathrm{ik}}}{R T} \\
G_{\mathrm{ji}}=\exp \left(-\alpha_{\mathrm{ji}} \tau_{\mathrm{ji}}\right) & \tau_{\mathrm{ji}}=\frac{\left(g_{\mathrm{ji}}-g_{\mathrm{ii}}\right)}{R T}=\frac{\Delta \mathrm{g}_{\mathrm{ji}}}{R T} \\
G_{\mathrm{jk}}=\exp \left(-\alpha_{\mathrm{jk}} \tau_{\mathrm{jk}}\right) & \tau_{\mathrm{jk}}=\frac{\left(g_{\mathrm{jk}}-g_{\mathrm{kk}}\right)}{R T}=\frac{\Delta \mathrm{g}_{\mathrm{jk}}}{R T} \\
G_{\mathrm{ki}}=\exp \left(-\alpha_{\mathrm{ki}} \tau_{\mathrm{ki}}\right) & \tau_{\mathrm{ki}}=\frac{\left(g_{\mathrm{ki}}-g_{\mathrm{ii}}\right)}{R T}=\frac{\Delta \mathrm{g}_{\mathrm{ki}}}{R T} \\
G_{\mathrm{kj}}=\exp \left(-\alpha_{\mathrm{kj}} \tau_{\mathrm{kj}}\right) & \tau_{\mathrm{kj}}=\frac{\left(g_{\mathrm{kj}}-g_{\mathrm{jj}}\right)}{R T}=\frac{\Delta \mathrm{g}_{\mathrm{kj}}}{R T}
\end{array}
$$

where $\Delta g_{\mathrm{ij}}, \Delta g_{\mathrm{ik}}, \Delta g_{\mathrm{ji}}, \Delta g_{\mathrm{jk}}, \Delta g_{\mathrm{ki}}$, and $\Delta g_{\mathrm{kj}}$ represent the cross interaction energy parameters which are independent of the composition and temperature. The 
non-random parameters $\alpha_{\mathrm{ij}}, \alpha_{\mathrm{ik}}, \alpha_{\mathrm{ji}}, \alpha_{\mathrm{jk}}, \alpha_{\mathrm{ki}}$ and $\alpha_{\mathrm{kj}}$ are empirical constants $\left(\alpha_{\mathrm{ij}}=\alpha_{\mathrm{ji}}\right.$, $\left.\alpha_{\mathrm{ik}}=\alpha_{\mathrm{ki}}, \alpha_{\mathrm{jk}}=\alpha_{\mathrm{kj}}\right)$, which generally varies between 0 and 1 .

\subsection{The CNIBS/R-K model}

The CNIBS/R-K model, expressed by Eq. (7), is suggested to be one of the best models to correlate the solubility in binary solvent systems [9].

$$
\ln x_{1}=x_{2}^{0} \ln \left(x_{1}\right)_{2}+x_{3}^{0} \ln \left(x_{1}\right)_{3}+x_{2}^{0} x_{3}^{0} \sum_{i=1}^{N} S_{i}\left(x_{2}^{0}-x_{3}^{0}\right)^{i}
$$

where $x_{1}$ is the mole fraction solubility of solute, while $x 02$ and $x 03$ represent the initial mole fraction composition of methanol and water in methanol-water mixtures, respectively; $\left(x_{1}\right)_{2}$ and $\left(x_{1}\right)_{3}$ are the mole fraction solubility of cefoxitin acid in pure methanol and water respectively; $S_{\mathrm{i}}$ is the model constant.

For binary solvent system, when $x 03$ is replaced by $(1-x 02)$ and $N=2$ is used, a general single model can be obtained as Eq. (8).

$$
\ln x_{1}=B_{0}+B_{1} x_{2}^{0}+B_{2}\left(x_{2}^{0}\right)^{2}+B_{3}\left(x_{2}^{0}\right)^{3}+B_{4}\left(x_{2}^{0}\right)^{4}
$$

where $B_{0}, B_{1}, B_{2}, B_{3}$ and $B_{4}$ are empirical parameters of the CNIBS/R-K model, which can be calculated by correlating the values of the experimental solubility.

\section{Results and discussion}

4.1. Identification and characterization of materials.

The PXRD patterns of cefoxitin acid anhydrate and its hydrate are shown in figure 2. It can be seen that cefoxitin acid anhydrate exhibit the characteristic peaks of $6.76^{\circ}$, 
$11.86^{\circ}, 12.32^{\circ}, 13.64^{\circ}, 14.73^{\circ}, 15.60^{\circ}, 17.98^{\circ}, 18.18^{\circ}, 18.66^{\circ}, 19.08^{\circ}, 19.86^{\circ}, 20.28^{\circ}$, $20.68^{\circ}, 21.26^{\circ}, 21.62^{\circ}, 22.46^{\circ}$ while the characteristic peaks of cefoxitin acid hydrate are at $12.42^{\circ}, 13.32^{\circ}, 16.27^{\circ}, 17.04^{\circ}, 18.86^{\circ}, 19.64^{\circ}, 20.00^{\circ}, 22.76^{\circ}, 24.54^{\circ}, 25.00^{\circ}$, $25.92^{\circ}, 26.20^{\circ}, 26.96^{\circ}, 27.86^{\circ}, 29.72^{\circ}$. In addition, the morphology of these two forms of cefoxitin acid (figure 3) shows that the crystal habit of cefoxitin acid anhydrate is rod-like while the crystal habit of cefoxitin acid hydrate is prism-like.

The TG/DSC data of the two forms were determined and plotted in figure 4. From the TG/DSC curves, it can be seen that cefoxitin acid anhydrate shows an exothermic peak and quick mass decreasing at $447.93 \mathrm{~K}$ (onset point $437.45 \mathrm{~K}$ ), which should represent its decomposition since the melting process should be endothermic. For cefoxitin acid hydrate, an endothermic peak at $374.38 \mathrm{~K}$ (onset point $355.43 \mathrm{~K}$ ) and an exothermic peak at $442.46 \mathrm{~K}$ (onset point $432.23 \mathrm{~K}$ ) were observed, respectively. It can also be seen that the sample weight of cefoxitin acid hydrate declines continuously from $355.43 \mathrm{~K}$ until it reaches a constant. Then at about $432.23 \mathrm{~K}$, the TG curve of cefoxitin acid hydrate declines sharply. The endothermic peak could be explained by the de-solvation of the solvent molecules in the crystal lattice and the exothermic peak should result from the decomposition of the dehydrated cefoxitin acid.

Furthermore, the ex situ Raman spectroscopy was also used to distinguish the differences between cefoxitin acid anhydrate and its hydrate. The characteristic peaks for these two forms are shown in figure 5. As shown in figure 5, there are some distinct differences between cefoxitin acid anhydrate and its hydrate. The peaks at 
$1716 \mathrm{~cm}^{-1}$ and $1792 \mathrm{~cm}^{-1}$ can be assigned as the characteristic peaks of cefoxitin acid anhydrate, while the peak at $1730 \mathrm{~cm}^{-1}$ and $1754 \mathrm{~cm}^{-1}$ can be assigned as the characteristic peaks of its hydrate. These differences can be used to distinguish them easily.

\subsection{Melting properties}

According to the study of Yalkowsky and Akash Jain [10-12], the enthalpy of melting $\left(\Delta_{\text {fus }} H\right)$ can be estimated by additive group contributions.

$\Delta_{\text {fus }} H=\sum n_{\mathrm{i}} m_{\mathrm{i}}+\sum n_{\mathrm{j}} m_{\mathrm{j}}$

where $n_{\mathrm{i}}$ and $n_{\mathrm{j}}$ respectively represent the number of times that group $\mathrm{i}$ and proximity factor $\mathrm{j}$ appears in a compound, $m_{\mathrm{i}}$ and $m_{\mathrm{j}}$ are the contribution of group $\mathrm{i}$ and proximity factor $\mathrm{j}$ to the enthalpy of melting, respectively.

The melting entropy $\left(\Delta_{\text {fus }} S\right)$ can be estimated by a semi-empirical equation with two non-additive molecular properties, rotational symmetry and flexibility. Bondi [13] has found that the melting entropy of a compound is the sum of its positional, rotational, and conformational components. On account of the very small volume change associated with melting process, the melting entropy of an organic molecule is primarily the contribution of its rotational and conformational entropies. Eq.(10) provides a method to estimate the entropy of melting.

$\Delta_{\text {fus }} S /\left(\mathrm{J} \cdot \mathrm{K}^{-1} \cdot \mathrm{mol}^{-1}\right)=50-8.3124 \ln \sigma+7.382 \tau$ 
where $\sigma=$ rotational symmetry number, $\tau=$ flexibility number. They account for the changes in geometrical restriction that accompany the process of melting. Since the molecular structure of cefoxitin acid has no symmetry, the $\sigma$ value of cefoxitin acid is 1. Dannenfelser [11] put forward that the flexibility number $\tau$ of a compound can be calculated using the following semi-empirical equation.

$\tau=S P 3+0.5 S P 2+0.5 R I N G-1$

where $S P 3=\Sigma$ Nonring $\left(\mathrm{CH}_{2}, \mathrm{CH}, \mathrm{C}, \mathrm{NH}, \mathrm{N}, \mathrm{O}, \mathrm{S}\right), S P 2=\Sigma$ Nonring $(=\mathrm{CH},=\mathrm{C},=\mathrm{N}$,

$\mathrm{C}=\mathrm{O}), R I N G=\Sigma$ Independent single, fused, or conjugated aromatic ring systems . According to the structure of cefoxitin acid, the value of $S P 3, S P 2$ and $R I N G$ are 12 , 10,3 , respectively.

By using the aforementioned method, the enthalpy and entropy of melting were estimated to be $71.58 \mathrm{~kJ} \cdot \mathrm{mol}^{-1}$ and $179.185 \mathrm{~J} \cdot \mathrm{K}^{-1} \cdot \mathrm{mol}^{-1}$, respectively. At the melting point, the Gibbs energy of transition is equal to zero and the melting temperature is estimated to be $399.475 \mathrm{~K}$ by the following Eq.(12).

$T_{\text {fus }}=\Delta_{\text {fus }} H / \Delta_{\text {fus }} S$

\subsection{Solubility values}

The UV calibration curves were obtained from the relationship between standard solution concentration and absorbance. For the standard solution, cefoxitin acid anhydrate was dissolved and diluted with the phosphate buffer (prepared by dissolving $1.0 \mathrm{~g}$ monobasic potassium phosphate and $1.8 \mathrm{~g}$ of anhydrous dibasic 
sodium phosphate in water to make $1000 \mathrm{~mL}$ ) or the organic solvents [5]. The function that relates the concentration of each solute with its absorbance was determined by fitting the absorbance data of standard solutions to a straight line. The calibration curves were shown in figure S1 of SUPPORTING INFORMATION. The values of the slope and intercept ([slope, intercept]) of calibration curves in methanol, ethanol, acetonitrile, ethyl acetate, isopropanol, n-propanol and phosphate buffer are determined to be $[0.01676,0.02354],[0.01579,0.01432],[0.01559,-0.00964]$, $[0.01535,0.0069],[0.01475,0.09179],[0.01449,0.03107]$ and $[0.02167,0.00126]$ with $\mathrm{R}^{2}=0.99909,0.99945,0.99986,0.99904,0.99981,0.99936$ and 0.99925 , respectively.

The PXRD patterns of the residual powder of cefoxitin acid in different solvents from $T=278.15 \mathrm{~K}$ to $T=308.15 \mathrm{~K}$ are given in figure $\mathrm{S} 2$ of SUPPORTING INFORMATION. As shown in figure 2 and figure S2, cefoxitin acid anhydrate was stable in pure organic solvent within the experimental temperature range. Cefoxitin acid hydrate was stable in pure water and water-methanol solvent mixtures when methanol mole fraction was less than 0.6. The water content of the residual cefoxitin acid crystal was determined via Karl Fischer titration, which was in accordance with the water content of the cefoxitin acid monohydrate.

The experimental solubility of cefoxitin acid in methanol, acetonitrile, ethanol, isopropanol, n-propanol, ethyl acetate are shown in figure 6 and table 2. It can be seen that the solubility of cefoxitin acid increases with rising temperature in all solvents investigated. At a given temperature, the order of cefoxitin acid anhydrate solubility is 
methanol $>$ ethanol $\approx$ acetonitrile $>$ n-propathenol $\approx$ isopropanol $\approx$ ethyl acetate. This phenomenon can be interpreted by hydrogen-bonding interaction and "like dissolves like" principle. According to the dielectric constant theory, the dielectric constant of solvent plays a very important role in solution, and the polarity of the material increases with the increasing of dielectric constant [14]. The order of the polarity of pure solvents used in this study is acetonitrile $>$ methanol $>$ ethanol $>$ n-propanol $>$ isopropanol > ethyl acetate. Three amides groups and one carboxyl group exist in the molecular structure of cefoxitin acid, which can act as H-bond donor and acceptor. Compared with methanol, acetonitrile has lower ability to form H-bond with cefoxitin acid and therefore has lower ability to dissolve cefoxitin acid. The solubility values of cefoxitin acid in water and water-methanol mixtures are shown in figure 7 and table 3. It is found that the solubility of cefoxitin acid increases as the mole fraction of methanol in the solvent mixture increases. This phenomenon can be explained by the fact that molecular interaction between cefoxitin acid and methanol molecules is stronger than the interaction between cefoxitin acid and water molecule. At the same composition of the binary solvent mixtures, the solubility of cefoxitin acid increases with increasing temperature. The solubility results can be used for designing and optimizing the process of crystallization of cefoxitin acid.

In this work, three thermodynamic models, including the modified Apelblat model, NRTL model and CNIBS/R-K model, were used to correlate the solubility of cefoxitin acid. The applicability of these models was evaluated by using the relative deviations (RD) and relative average deviations (ARD). The RD and ARD are defined as Eqs. 
(13) and (14) [15].

$\mathrm{RD}=\frac{x_{1, i}^{\mathrm{exp}}-x_{1, i}^{\mathrm{cal}}}{x_{1, i}^{\exp }}$

$\mathrm{ARD}=\frac{1}{n} \sum_{i=1}^{n}\left|\frac{x_{1, i}^{\text {exp }}-x_{1, i}^{\mathrm{cal}}}{x_{1, i}^{\exp }}\right|$

Here $x \exp 1, \mathrm{i}$ and $x$ cal $1, \mathrm{i}, \mathrm{i}$ are experimental solubility and calculated solubility, respectively; $n$ is the number of experimental points. The calculated results of RD and ARD are shown in tables 2 to 8 . The model parameters obtained for cefoxitin acid in the organic solvents are shown in tables 4 and 5 while the model parameters obtained for cefoxitin acid in water and water-methanol mixtures are shown in tables 6 to 8 . From tables 2 to 8 , it is found that the correlated results by the modified Apelblat equation, NRTL model and CNIBS/R-K model show good consistency with the experimental solubility with ARD values less than $8 \%$ and these models are all suitable for correlating the solubility of cefoxitin acid in the selected solvents. Obviously, the modified Apelblat equation can give better correlation results with ARD values less than $5 \%$.

\subsection{The calculation of dissolution enthalpy}

Following the rigorous thermodynamic treatment of the solubility of solutes (electrolytes and nonelectrolytes) in solvents given by Williamson $[16,17,18]$, the molar enthalpy of solution $\Delta_{\text {sol }} H$ can be determined by, 


$$
\Delta H_{\mathrm{sol}}=v\left(1-\frac{h m_{1} M_{2}}{1000}\right) R T^{2}\left(\frac{\mathrm{d} m_{1}}{\mathrm{~d} T}\right)_{\mathrm{sat}}\left[\left(\frac{\partial \ln a_{2}}{\partial m_{1}}\right)_{T, \mathrm{sat}}\right]
$$

In this precise equation, $\Delta_{\text {sol }} H$ is the enthalpy absorbed per mole of solute dissolved in the nearly saturated solution, or the enthalpy evolved per mole on crystallisation. The $v$ denotes the total number of ions formed by one molecule of solute, and $v=1$ is introduced for nonelectrolytes, $h$ is the hydration number, $m_{1}$ is the molality of the solute, $R$ is the gas constant and $M_{2}$ and $a_{2}$ are the molecular mass and activity of the solvent, respectively. In terms of experimentally available mean activity coefficients $\gamma_{ \pm}$, Eq.(15) becomes,

$$
\Delta H_{\text {sol }}=v\left(1-\frac{h m_{1} M_{2}}{1000}\right) R T^{2}\left(\frac{\mathrm{d} m_{1}}{\mathrm{~d} T}\right)_{s a t}\left[\left(\frac{\partial \ln \gamma_{ \pm}}{\partial m_{1}}\right)_{\text {sat }, T}+\left(\frac{1}{m_{1}}\right)_{\text {sat }}\right]
$$

In the case of nonelectrolytes, the mean activity coefficient is replaced by the activity coefficient of the solute $\left(\gamma_{1}\right)$. Beside, $m_{1}=\frac{1000 x_{1}}{\left(1-x_{1}\right) M_{2}}\left(M_{2}\right.$ and $x_{1}$ are the molecular mass of the solvent and mole fraction of the solute, respectively) and, in the case of this work, $x_{1} \ll<1$. Therefore, $\Delta H_{\text {sol }}$ can be calculated by

$$
\Delta H_{\mathrm{sol}}=v\left(1-\frac{h x_{1}}{1-x_{1}}\right) R T^{2}\left[\left(\frac{\mathrm{d} \ln x_{1}}{\mathrm{~d} T}\right)_{s a t}+\left(\frac{\mathrm{d} x_{1}}{\mathrm{~d} T}\right)_{s a t}\left(\frac{\partial \ln \gamma_{1}}{\partial x_{1}}\right)_{\mathrm{sat}, T}\right]
$$

For cefoxitin acid, $v=1 . h=0$ and 1 represents anhydrate and hydrate of cefoxitin acid, respectively. The terms $\left(\frac{\mathrm{d} \ln x_{1}}{\mathrm{~d} T}\right)_{\text {sat }}$ and $\left(\frac{\mathrm{d} x_{1}}{\mathrm{~d} T}\right)_{\text {sat }}$ can be calculated from Eq. (3) and $\left(\frac{\partial \ln \gamma_{1}}{\partial x_{1}}\right)_{\text {sat }, T}$ can be calculated by Eq. (5). The calculated results of $\Delta_{\text {sol }} H$ of the dissolution process are listed in table 9 and table 10. It is found that the values of $\Delta_{\text {sol }} H$ are all positive in the solvents investigated, indicating that the dissolution process is endothermic. 


\section{Conclusions}

The solubility of cefoxitin acid in six pure organic solvents (methanol, acetonitrile, ethanol, isopropanol, n-propanol and ethyl acetate), pure water and water-methanol mixtures was measured using a UV method within the temperature range $278.15 \mathrm{~K}$ to 303.15 K. The PXRD data and Karl Fischer method were used to verify the crystal form stability of cefoxitin acid in the solubility measuring process. The melting enthalpy was estimated from group contributions as $71.58 \mathrm{~kJ} \cdot \mathrm{mol}^{-1}$, whereas the melting entropy was estimated using a semi-empirical equation as $179.185 \mathrm{~J} \cdot \mathrm{K}^{-1} \cdot \mathrm{mol}^{-1}$. The melting temperature was calculated from the ratio of the enthalpy and entropy of melting as $399.475 \mathrm{~K}$. Results shows that the solubility of cefoxitin acid increases with rising temperature in all solvents used in this work. The order of solubility of cefoxitin acid anhydrate is methanol $>$ ethanol $\approx$ acetonitrile $>$ n-propanol $\approx$ isopropanol $\approx$ ethyl acetate. This phenomenon can be interpreted by hydrogen-bonding interaction and "like dissolves like" principle. The solubility of cefoxitin acid in water and water-methanol mixtures increases with increasing methanol concentration. The modified Apelblat equation and NRTL model were used to correlate the experimental solubility values of cefoxitin acid in pure organic solvents while the CNIBS/R-K model was also utilized to correlate the solubility of cefoxitin acid in water and methanol-water solvents. All the selected thermodynamic models gave satisfactory correlation results. The dissolution enthalpy was precisely calculated by the equation put forward by Williamson. 


\section{Acknowledgement}

The authors are very thankful to the National Science Foundation of China (No. 21376165) and the Key Project of Tianjin Science and Technology Supporting Program (No. 13ZCZDNC02000) for the financial support.

\section{References}

[1] J. Birnbaum, E. Stapley, A. Miller, H. Wallick, D. Hendlin, H. Woodruff, Journal of Antimicrobial Chemotherapy. 4 (1978) 15-32.

[2] M. Gurwith, W. Albritton, B. Lank, G. Harding, A. Ronald, Journal of Antimicrobial Chemotherapy. 4 (1978) 211-213.

[3] H. Kaemmerer, M.J. Jones, H. Lorenz, A. Seidel-Morgenstern, Fluid Phase Equilibria. 296 (2010) 192-205.

[4] M.W. Hermanto, P.S. Chow, R.B. Tan, Industrial \& Engineering Chemistry Research. 51 (2012) 13773-13783.

[5] U.S. Pharmacopeia National Formulary. USP 35 NF 30. The United States Pharmacopeial Convention, 2012, Volume 1: 2563-2564.

[6] E.R. Oberholtzer, G.S. Brenner, Journal of pharmaceutical sciences. 68 (1979) 863-866.

[7] A. Apelblat, E. Manzurola, The Journal of Chemical Thermodynamics. 31 (1999) $85-91$

[8] K Zhao, L Lin, C. Li, S, Du, C Huang, Y, Qin, P Yang, K Li, J Gong, Journal of Chemical Thermodynamic. 61 (2016) 1210-1220.

[9] W.E. Acree, Thermochimica Acta. 198 (1992) 71-79. 
[10] R.M. Dannenfelser, S.H. Yalkowsky, Industrial \& Engineering Chemistry Research. 35 (1996) 1483-1486.

[11] P. Simamora, S.H. Yalkowsky, Industrial \& Engineering Chemistry Research. 33 (1994) 1405-1409.

[12] A. Jain, G. Yang, S.H. Yalkowsky, Industrial \& Engineering Chemistry Research. 43 (2004) 7618-7621.

[13] A.A. Bondi, Science. 163 (1969) 429.

[14] J.G. Speight, Lange's handbook of chemistry, New York. 2005.

[15] C. Huang, Z. Xie, J. Xu, Y. Qin, Y. Du, S. Du, J. Gong, Journal of Chemical \& Engineering Data. 60 (2015) 870-875.

[16] A.T. Williamson, Trans. Farad. Soc. 40 (1944) 421-436.

[17] J. Cuevas-Valenzuela, Á. González-Rojas, J. Wisniak, A. Apelblat, J.R. Pérez-Correa, Fluid Phase Equilibria. 382 (2014) 279-285.

[18] A. Apelblat, E. Manzurola, N.A. Balal, J. Chem. Thermodynamics. 38 (2006) $565-571$.

\section{Figure captions:}

Figure 1. Chemical structure of cefoxitin acid.

Figure 2. X-ray power diffraction pattern of cefoxitin acid anhydrate (a) and hydrate (b). 
Figure 3. Microscope pictures of cefoxitin acid anhydrate (a) and its hydrate (b).

Figure 4. Thermal analysis (TGA/DSC) of cefoxitin acid. The TGA curves of cefoxitin acid anhydrate (a) and hydrate (b), and the DSC curves of cefoxitin acid anhydrate (c) and hydrate (d).

Figure 5 Raman spectra of cefoxitin acid anhydrate (red) and its hydrate (blue).

Figure 6. Experimental solubility values of cefoxitin acid in methanol (घ), n-propanol $(\boldsymbol{\nabla})$, isopropanol $(\boldsymbol{\bullet})$, ethanol $(\boldsymbol{\nabla})$, acetonitrile $(\boldsymbol{\Delta})$, ethyl acetate $(\bullet)$, Calculated solubilities with Eq.(3) for cefoxitin acid (solid line) are also included.

Figure 7. Experimental solubility values of cefoxitin acid in water ( $\mathbf{a})$ and water-methanol mixtures $(x 02=0.1013(\bullet), 0.2011(\mathbf{\Delta}), 0.2933(\boldsymbol{\nabla}), 0.4020(\varangle)$, $0.5003(\triangleright), 0.5993(\diamond))$. Calculated solubility with Eq.(3) for cefoxitin acid in water and methanol-water mixtures (solid line) are also included.

\section{Table headings:}

Table 1 Provenance and mass fraction purity of cefoxitin acid and solvents.

Table 2 Experimental and correlated mole fraction solubility of cefoxitin acid $\left(x_{1}{ }^{\exp }\right.$ and $x_{1}{ }^{\mathrm{cal}}$ ) in methanol, acetonitrile, ethanol, isopropanol, n-propanol and ethyl acetate $(p=0.1 \mathrm{MPa})$

Table 3 Experimental and correlated mole fraction solubility of cefoxitin acid ( $x_{1}{ }^{\exp }$ and $x_{1}{ }^{\mathrm{cal}}$ ) in water and (methanol + water) mixtures at different initial mole fractions of methanol $(x 02)$ and different temperatures $(p=0.1 \mathrm{MPa})$.

Table 4 Parameters of the modified Apelblat equation for the solubility of cefoxitin 
acid in methanol, acetonitrile, ethanol, isopropanol, n-propanol and ethyl acetate.

Table 5 Parameters of the NRTL model for the solubility of cefoxitin acid in methanol, acetonitrile, ethanol, isopropanol, n-propanol and ethyl acetate.

Table 6 Parameters of the modified Apelblat equation for the solubility of cefoxitin acid in water and (methanol + water) mixtures at different initial mole fractions of methanol ( $x 0$ 2).

Table 7 Parameters of the NRLT model for the solubility of cefoxitin acid in water and (methanol + water) mixtures.

Table 8 Parameters of the CNIBS/R-K model for the solubility of cefoxitin acid in water and (methanol + water) mixtures.

Table 9 Molar enthalpy $\left(\Delta_{\text {sol }} H\right)$ of the cefoxitin acid dissolution process in methanol, acetonitrile, ethanol, isopropanol, n-propanol and ethyl acetate.

Table 10 Molar enthalpy $\left(\Delta_{\text {sol }} H\right)$ of the cefoxitin acid dissolution process in water and (methanol + water) mixtures at different initial mole fractions of methanol $(x 02)$.

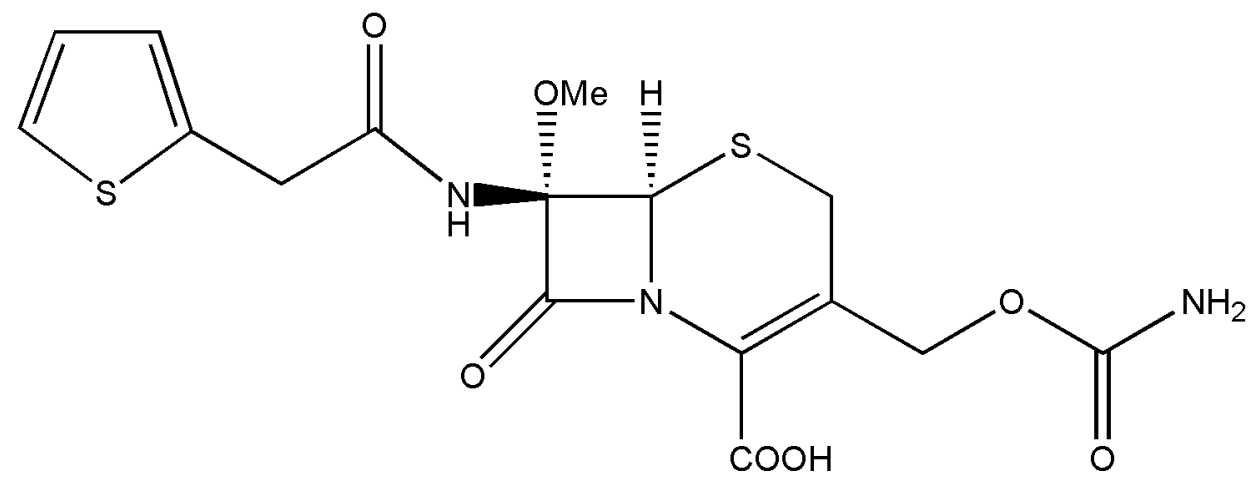

Figure 1. Chemical structure of cefoxitin acid. 


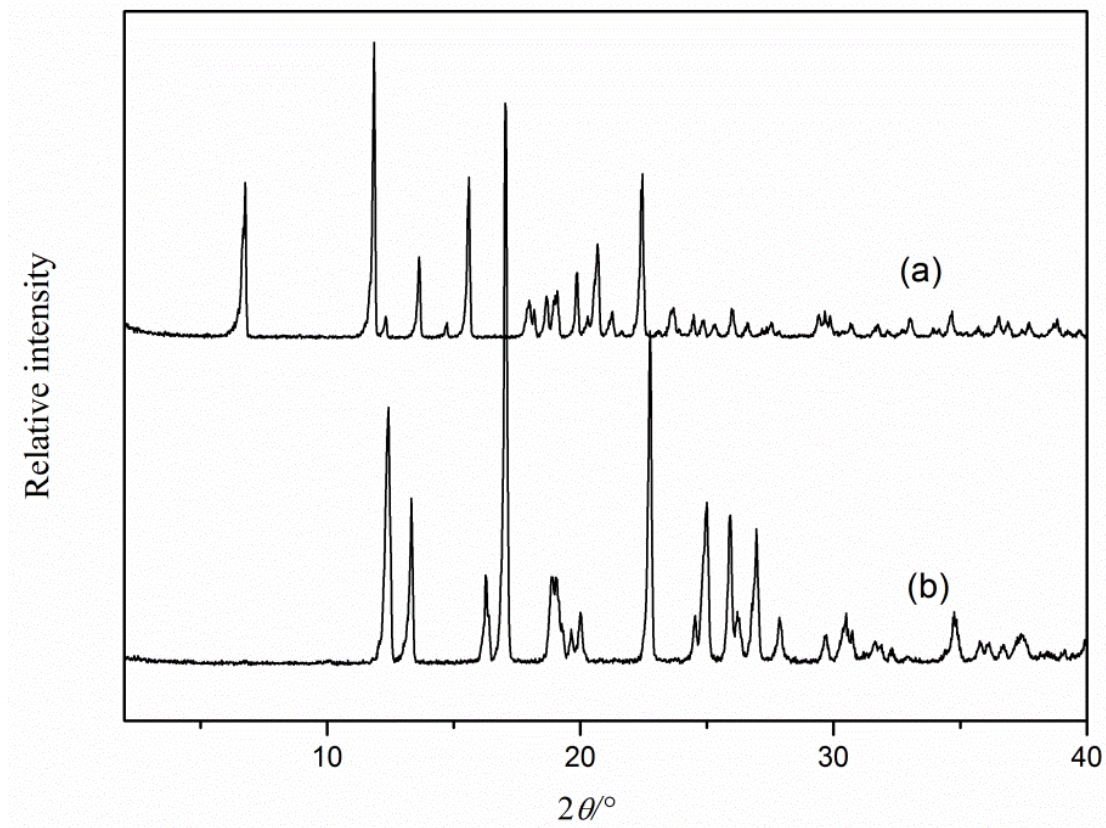

Figure 2. X-ray power diffraction patterns of cefoxitin acid anhydrate (a) and hydrate (b).
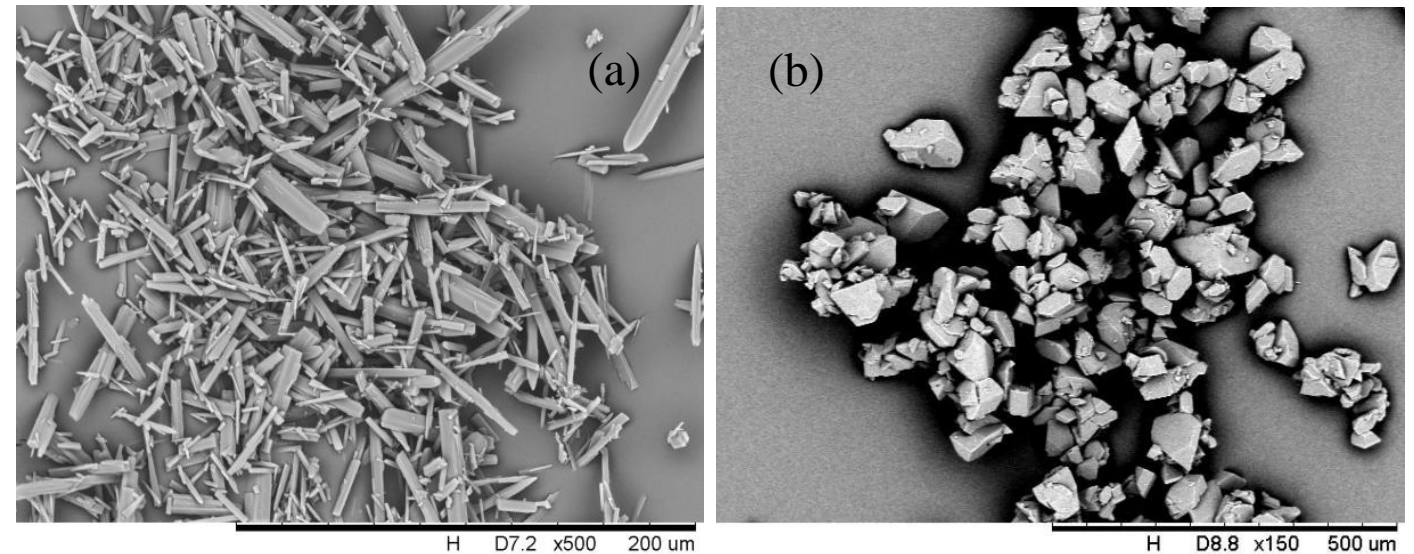

Figure 3. Microscope pictures of cefoxitin acid anhydrate (a) and its hydrate (b). 


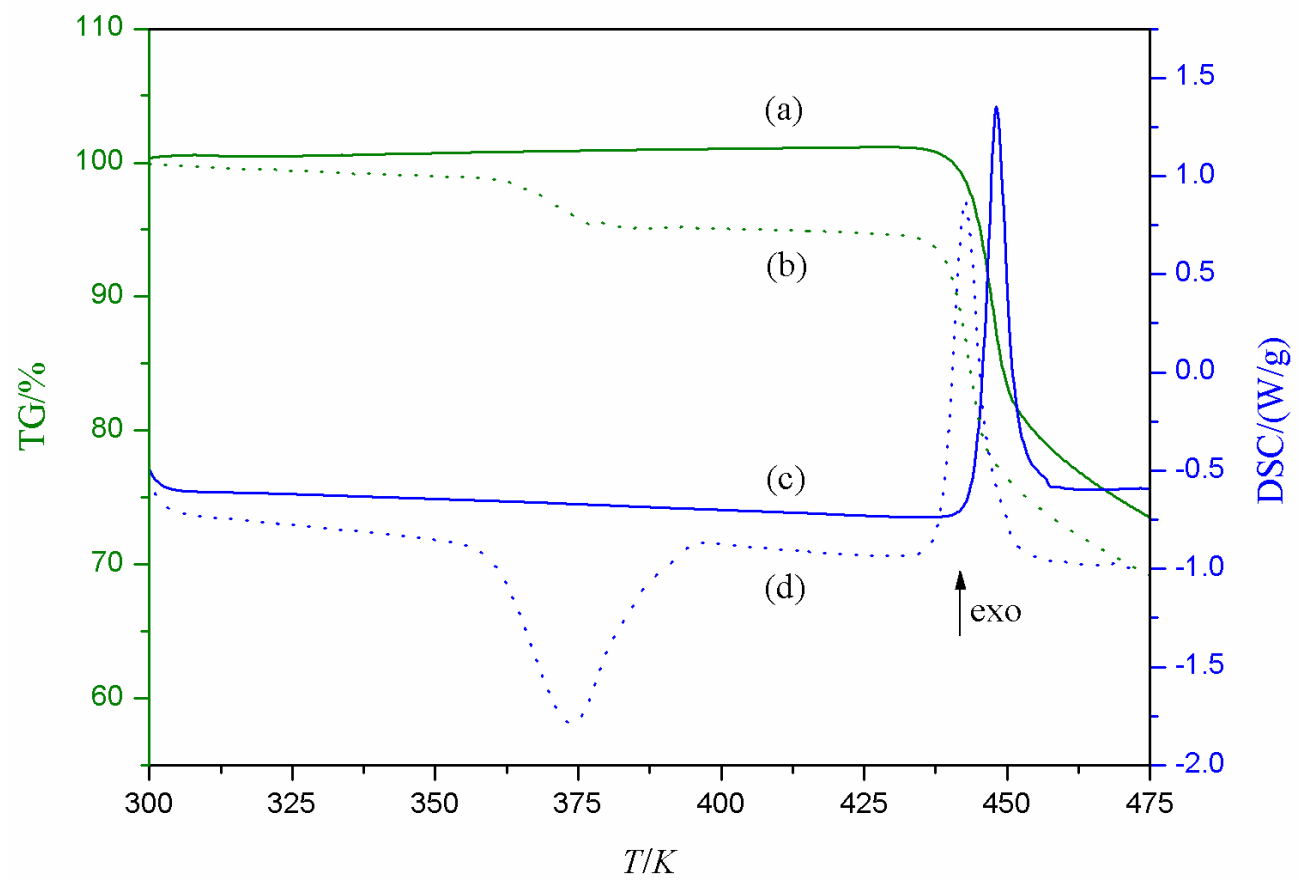

Figure 4. Thermal analysis (TGA/DSC) of cefoxitin acid. The TGA curves of cefoxitin acid anhydrate (a) and hydrate (b), and the DSC curves of cefoxitin acid anhydrate (c) and hydrate (d).

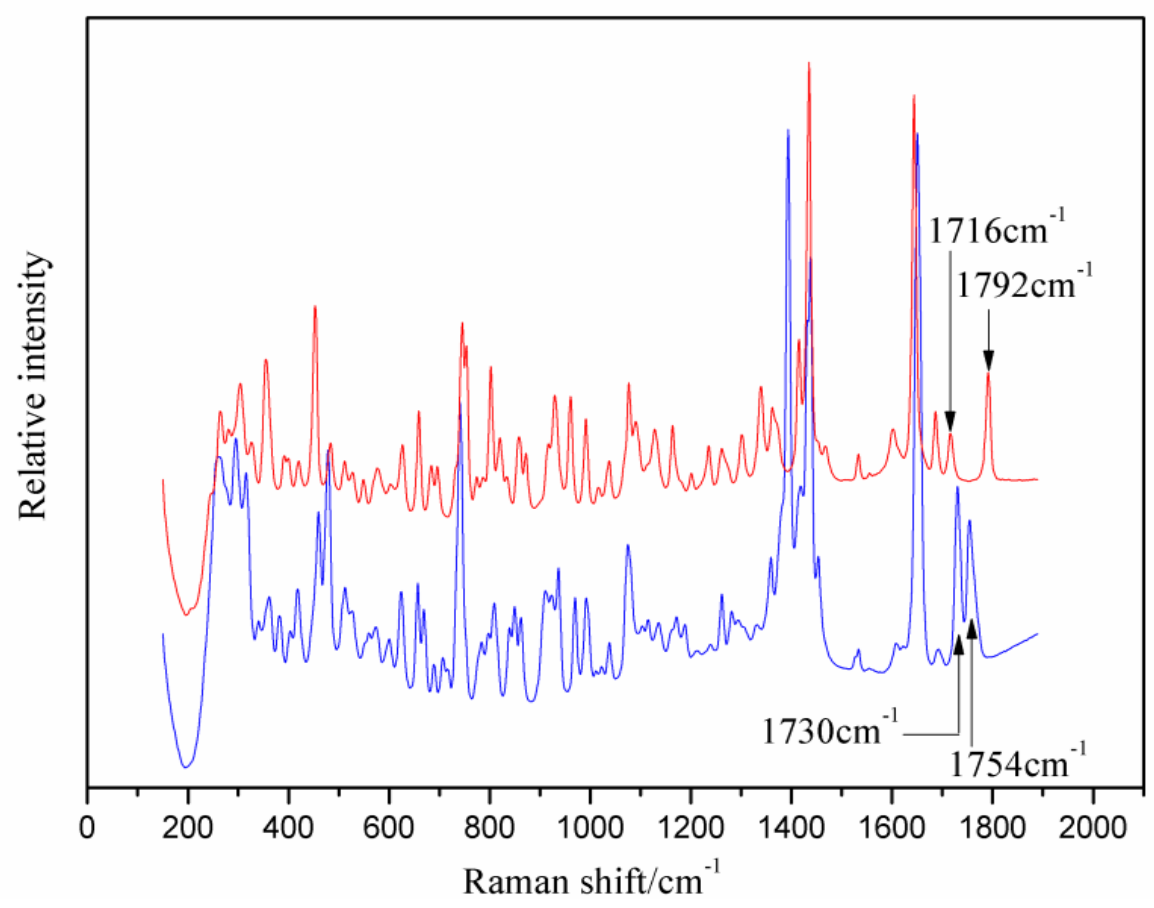

Figure 5. Raman spectra of cefoxitin acid anhydrate (red) and its hydrate (blue). 


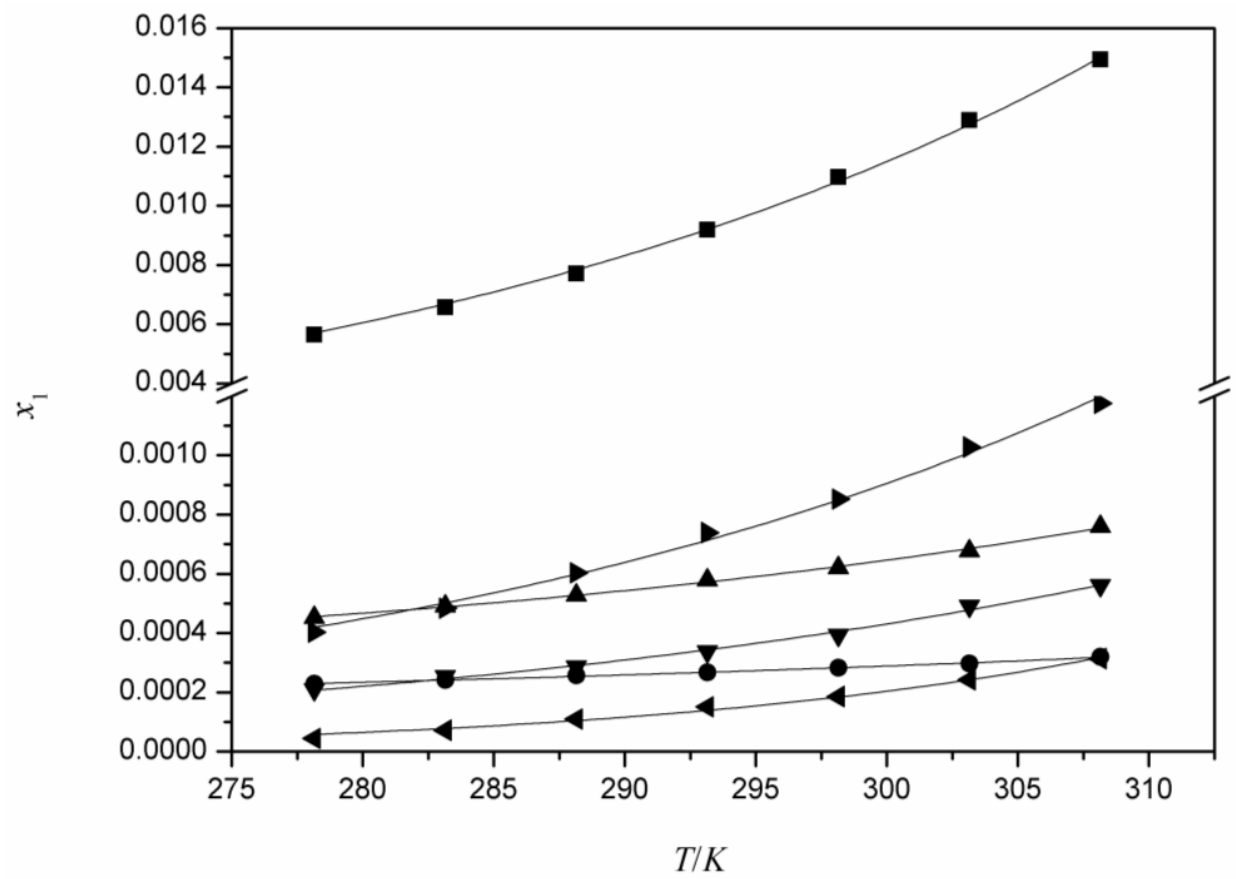

Figure 6. Experimental solubilities of cefoxitin acid in methanol ( $\mathbf{\square})$, n-propanol ( $\boldsymbol{\nabla})$, isopropanol $(\boldsymbol{\bullet})$, ethanol $(\boldsymbol{\bullet})$, acetonitrile $(\boldsymbol{\Delta})$, ethyl acetate $(\bullet)$. Calculated solubilities with Eq.(3) for cefoxitin acid (solid line) are also included.

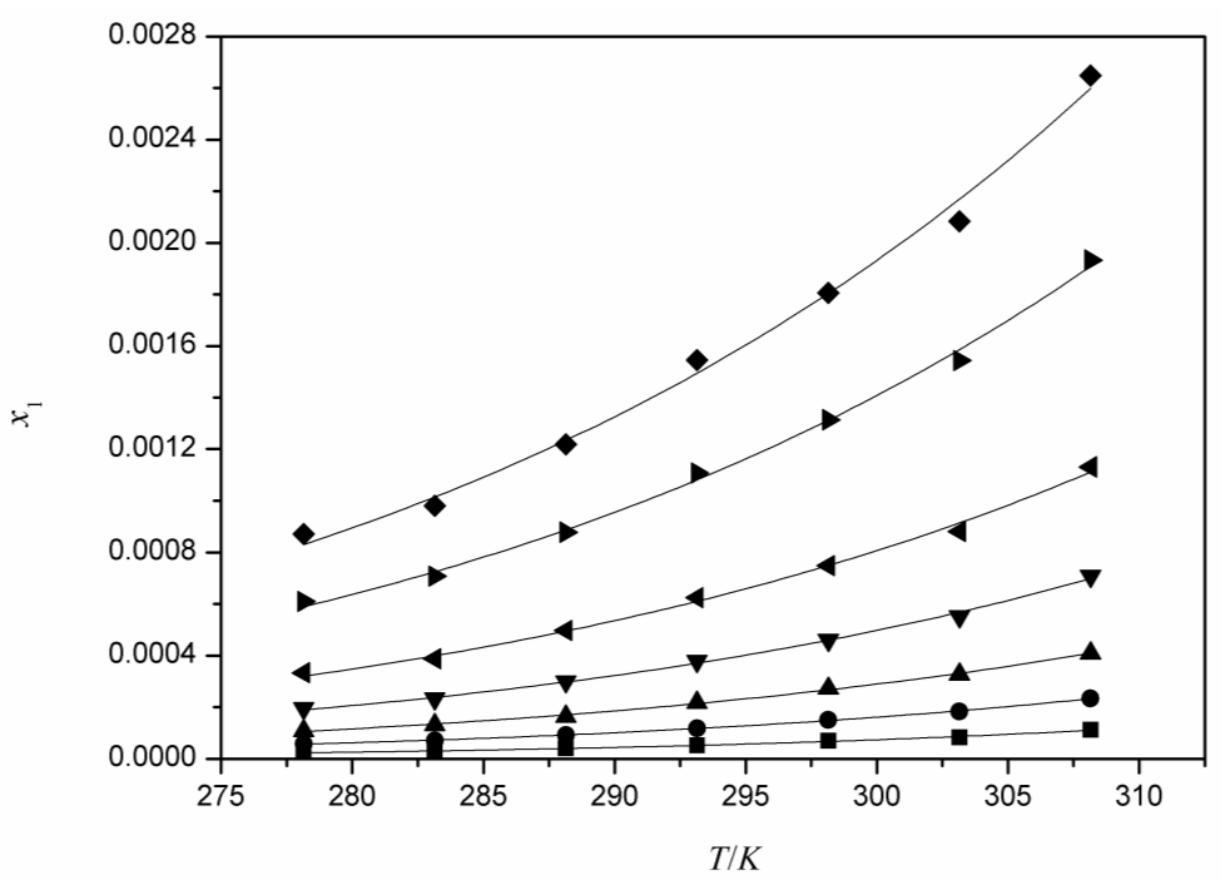


Figure 7. Experimental solubilities of cefoxitin acid in water (ש) and water-methanol

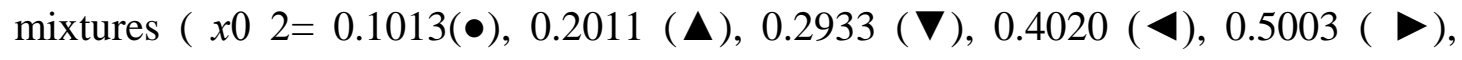
$0.5993(\diamond))$. Calculated solubility using Eq.(3) for cefoxitin acid in water and methanol-water mixtures (solid line) are also included.

\section{Table 1}

Provenance and mass fraction purity of cefoxitin acid and solvents. ${ }^{a, b}$

\begin{tabular}{|c|c|c|c|c|}
\hline Chemicals & $\begin{array}{l}\text { Mass } \\
\text { purity }\end{array}$ & $\begin{array}{l}\text { Method of } \\
\text { Purification }\end{array}$ & $\begin{array}{c}\text { Analysis } \\
\text { method }\end{array}$ & Source \\
\hline $\begin{array}{l}\text { Cefoxitin acid } \\
\text { monohydrate }\end{array}$ & $\geq 0.995$ & None & $\mathrm{HPLC}^{a}$ & $\begin{array}{c}\text { Hebei Huamin } \\
\text { Pharmaceutical Co., Ltd. }\end{array}$ \\
\hline $\begin{array}{l}\text { Cefoxitin acid } \\
\text { anhydrate }\end{array}$ & $\geq 0.998$ & None & HPLC $^{a}$ & Lab-Synthesis \\
\hline Methanol & $\geq 0.995$ & None & $\mathrm{GC}^{b}$ & $\begin{array}{c}\text { Tianjin Jiangtian Chemical } \\
\text { Co., Ltd. }\end{array}$ \\
\hline Ethanol & $\geq 0.995$ & none & $\mathrm{GC}^{b}$ & $\begin{array}{c}\text { Tianjin Jiangtian Chemical } \\
\text { Co., Ltd. }\end{array}$ \\
\hline n-propanol & $\geq 0.995$ & none & $\mathrm{GC}^{b}$ & $\begin{array}{c}\text { Tianjin Jiangtian Chemical } \\
\text { Co., Ltd. }\end{array}$ \\
\hline Isopropanol & $\geq 0.995$ & none & $\mathrm{GC}^{b}$ & $\begin{array}{c}\text { Tianjin Jiangtian Chemical } \\
\text { Co., Ltd. }\end{array}$ \\
\hline Ethyl acetate & $\geq 0.995$ & none & $\mathrm{GC}^{b}$ & $\begin{array}{c}\text { Tianjin Jiangtian Chemical } \\
\text { Co., Ltd. }\end{array}$ \\
\hline Acetonitrile & $\geq 0.995$ & none & $\mathrm{GC}^{b}$ & $\begin{array}{c}\text { Tianjin Jiangtian Chemical } \\
\text { Co., Ltd. }\end{array}$ \\
\hline
\end{tabular}

${ }^{a}$ High-performance liquid chromatography;

${ }^{b}$ Gas-liquid chromatography.

\section{Table 2}

Experimental and correlated mole fraction solubilities of cefoxitin acid ( $x_{1}{ }^{\exp }$ and $\left.x_{1}{ }^{\mathrm{cal}}\right)$ in methanol, acetonitrile, ethanol, isopropanol, n-propanol and ethyl acetate ( $p=0.1$ $\mathrm{MPa})^{a, b, c, d, e}$ 


\begin{tabular}{|c|c|c|c|c|c|}
\hline $\mathrm{T} / \mathrm{K}$ & $10^{4} x_{1}{ }^{\exp }$ & $10^{4} x_{1}{ }^{\mathrm{cal}}$ & $10^{2} \mathrm{RD}$ & $10^{4} x_{1}{ }^{\mathrm{cal}}$ & $10^{2} \mathrm{RD}$ \\
\hline \multicolumn{6}{|c|}{ Methanol } \\
\hline 278.15 & 56.46 & 55.60 & 1.5 & 59.48 & -5.3 \\
\hline 283.15 & 65.75 & 66.05 & -0.45 & 66.84 & -1.7 \\
\hline 288.15 & 77.17 & 78.24 & -1.4 & 76.50 & 0.87 \\
\hline 293.15 & 91.94 & 92.43 & -0.53 & 89.31 & 2.9 \\
\hline 298.15 & 109.7 & 108.9 & 0.71 & 106.0 & 3.4 \\
\hline 303.15 & 128.9 & 128.0 & 0.67 & 127.1 & 1.4 \\
\hline 308.15 & 149.5 & 150.1 & -0.42 & 153.8 & -2.9 \\
\hline \multicolumn{6}{|c|}{ Ethyl acetate } \\
\hline 278.15 & 2.289 & 2.3 & -0.49 & 2.386 & -4.2 \\
\hline 283.15 & 2.412 & 2.4 & -0.064 & 2.396 & 0.65 \\
\hline 288.15 & 2.571 & 2.5 & 1.3 & 2.464 & 4.2 \\
\hline 293.15 & 2.668 & 2.7 & -0.29 & 2.584 & 3.2 \\
\hline 298.15 & 2.825 & 2.8 & -0.087 & 2.762 & 2.3 \\
\hline 303.15 & 2.964 & 3.0 & -1.0 & 2.998 & -1.1 \\
\hline 308.15 & 3.198 & 3.2 & 0.62 & 3.305 & -3.3 \\
\hline \multicolumn{6}{|c|}{ Acetonitrile } \\
\hline 278.15 & 4.521 & 4.6 & -0.78 & 4.708 & -4.1 \\
\hline 283.15 & 4.914 & 4.9 & 0.57 & 4.895 & 0.39 \\
\hline 288.15 & 5.278 & 5.3 & 0.10 & 5.189 & 1.7 \\
\hline 293.15 & 5.795 & 5.7 & 1.2 & 5.605 & 3.3 \\
\hline 298.15 & 6.206 & 6.2 & -0.66 & 6.142 & 1.0 \\
\hline 303.15 & 6.773 & 6.9 & -1.2 & 6.830 & -0.84 \\
\hline 308.15 & 7.602 & 7.6 & 0.68 & 7.700 & -1.3 \\
\hline \multicolumn{6}{|c|}{ n-propanol } \\
\hline 278.15 & 2.092 & 2.1 & -0.86 & 2.073 & 0.88 \\
\hline 283.15 & 2.534 & 2.5 & 2.8 & 2.400 & 5.3 \\
\hline 288.15 & 2.858 & 2.9 & -1.1 & 2.810 & 1.7 \\
\hline 293.15 & 3.375 & 3.4 & -0.81 & 3.325 & 1.5 \\
\hline 298.15 & 3.935 & 4.0 & -2.2 & 3.970 & -0.90 \\
\hline 303.15 & 4.917 & 4.8 & 3.0 & 4.786 & 2.7 \\
\hline 308.15 & 5.613 & 5.7 & -1.0 & 5.795 & -3.2 \\
\hline \multicolumn{6}{|c|}{ Isopropanol } \\
\hline 278.15 & 0.440 & 0.5 & -13 & 0.530 & -21 \\
\hline 283.15 & 0.719 & 0.7 & -1.8 & 0.719 & 0.0088 \\
\hline 288.15 & 1.094 & 1.0 & 4.7 & 0.975 & 11 \\
\hline 293.15 & 1.508 & 1.4 & 4.9 & 1.322 & 12 \\
\hline 298.15 & 1.858 & 1.9 & -2.9 & 1.790 & 3.7 \\
\hline 303.15 & 2.428 & 2.5 & -1.9 & 2.420 & 0.30 \\
\hline 308.15 & 3.138 & 3.1 & 0.9 & 3.268 & -4.1 \\
\hline \multicolumn{6}{|c|}{ Ethanol } \\
\hline 278.15 & 4.034 & 4.0 & 1.6 & 4.180 & -3.6 \\
\hline 283.15 & 4.838 & 4.9 & -2.0 & 5.012 & -3.6 \\
\hline
\end{tabular}




\begin{tabular}{lccccc}
288.15 & 6.029 & 6.0 & -0.16 & 5.992 & 0.60 \\
293.15 & 7.389 & 7.3 & 1.4 & 7.146 & 3.3 \\
298.15 & 8.521 & 8.7 & -1.6 & 8.505 & 0.19 \\
303.15 & 10.28 & 10.2 & 1.1 & 10.09 & 1.8 \\
308.15 & 11.76 & 11.8 & -0.31 & 11.96 & -1.8 \\
\hline
\end{tabular}

${ }^{a}$ The reported results were the average values of three determinations;

${ }^{b}$ The standard uncertainty of temperature is $u(T)=0.03 \mathrm{~K}$;

${ }^{c}$ The relative standard uncertainty of the solubility measurement is $u_{\mathrm{r}}\left(x_{1}\right)=0.05$; the standard uncertainty of the pressure is $u(p)=10 \mathrm{kPa}$;

${ }^{d} \mathrm{RD}$ is the relative deviation;

${ }^{e}$ The residual solid phase of cefoxitin acid in the solubility experiments was anhydrous cefoxitin acid.

\section{Table 3}

Experimental and correlated mole fraction solubilities of cefoxitin acid $\left(x_{1}{ }^{\text {exp }}\right.$ and $\left.x_{1}{ }^{\mathrm{cal}}\right)$ in water and (methanol + water) mixtures at different initial mole fractions of methanol ( $x 02$ 2) and different temperatures $(p=0.1 \mathrm{MPa}){ }^{a, b, c, d, e, f}$

\begin{tabular}{|c|c|c|c|c|c|c|c|c|}
\hline \multirow[b]{2}{*}{$T / \mathrm{K}$} & \multirow[b]{2}{*}{$10 x_{2}$} & \multirow[b]{2}{*}{$10^{5} x_{1}^{\exp }$} & \multicolumn{2}{|c|}{ Apelblat } & \multicolumn{2}{|c|}{ NRTL } & \multicolumn{2}{|c|}{ CNIBS/R-K } \\
\hline & & & $10^{5} x_{1}{ }^{\mathrm{cal}}$ & $10^{2} \mathrm{RD}$ & $10^{5} x_{1}{ }^{\mathrm{cal}}$ & $10^{2} \mathrm{RD}$ & $10^{5} x_{1}{ }^{\mathrm{cal}}$ & $10^{2} \mathrm{RD}$ \\
\hline \multicolumn{9}{|c|}{ Water } \\
\hline 278.15 & 0.0000 & 2.460 & 2.453 & 0.30 & 2.883 & -17 & 1.893 & 23 \\
\hline 283.15 & 0.0000 & 3.028 & 3.120 & -3.0 & 3.780 & -25 & 2.356 & 22 \\
\hline 288.15 & 0.0000 & 4.025 & 3.991 & 0.85 & 4.930 & -22 & 3.399 & 16 \\
\hline 293.15 & 0.0000 & 5.127 & 5.130 & -0.05 & 6.399 & -25 & 4.132 & 19 \\
\hline 298.15 & 0.0000 & 6.918 & 6.623 & 4.3 & 8.268 & -20 & 5.723 & 17 \\
\hline 303.15 & 0.0000 & 8.227 & 8.586 & -4.4 & 10.64 & -29 & 6.740 & 18 \\
\hline 308.15 & 0.0000 & 11.29 & 11.17 & 1.0 & 13.62 & -21 & 9.753 & 14 \\
\hline \multicolumn{9}{|c|}{$x 02=0.1013$} \\
\hline 278.15 & 1.0129 & 5.674 & 5.613 & 1.1 & 5.256 & 7.4 & 6.029 & -6.3 \\
\hline 283.15 & 1.0129 & 7.130 & 7.232 & -1.4 & 6.791 & 4.7 & 7.537 & -5.7 \\
\hline 288.15 & 1.0128 & 9.227 & 9.251 & -0.26 & 8.736 & 5.3 & 9.554 & -3.5 \\
\hline 293.15 & 1.0128 & 11.76 & 11.76 & 0.0002 & 11.19 & 4.8 & 12.43 & -5.8 \\
\hline 298.15 & 1.0127 & 15.09 & 14.84 & 1.7 & 14.27 & 5.4 & 16.07 & -6.5 \\
\hline 303.15 & 1.0126 & 18.36 & 18.62 & -1.4 & 18.13 & 1.2 & 19.50 & -6.3 \\
\hline 308.15 & 1.0125 & 23.30 & 23.22 & 0.33 & 22.95 & 1.5 & 24.54 & -5.3 \\
\hline \multicolumn{9}{|c|}{$x 02=0.2011$} \\
\hline 278.15 & 2.0106 & 10.86 & 10.49 & 3.4 & 9.848 & 9.3 & 11.39 & -4.9 \\
\hline 283.15 & 2.0105 & 13.18 & 13.43 & -1.9 & 12.54 & 4.9 & 13.86 & -5.1 \\
\hline 288.15 & 2.0103 & 16.48 & 17.05 & -3.5 & 15.90 & 3.5 & 17.50 & -6.2 \\
\hline 293.15 & 2.0101 & 21.77 & 21.46 & 1.4 & 20.09 & 7.7 & 22.74 & -4.4 \\
\hline
\end{tabular}




\begin{tabular}{|c|c|c|c|c|c|c|c|c|}
\hline 298.15 & 2.0099 & 27.29 & 26.80 & 1.8 & 25.30 & 7.3 & 28.26 & -3.5 \\
\hline 303.15 & 2.0097 & 32.79 & 33.21 & -1.3 & 31.75 & 3.2 & 34.09 & -4.0 \\
\hline 308.15 & 2.0094 & 40.94 & 40.86 & 0.19 & 39.71 & 3.0 & 42.85 & -4.7 \\
\hline \multicolumn{9}{|c|}{$x 02=0.2933$} \\
\hline 278.15 & 2.9319 & 19.49 & 19.39 & 0.52 & 17.75 & 8.9 & 18.35 & 5.8 \\
\hline 283.15 & 2.9316 & 23.23 & 23.99 & -3.3 & 22.29 & 4.1 & 21.78 & 6.2 \\
\hline 288.15 & 2.9312 & 29.87 & 29.72 & 0.50 & 27.89 & 6.6 & 28.01 & 6.2 \\
\hline 293.15 & 2.9308 & 37.87 & 36.83 & 2.7 & 34.80 & 8.1 & 35.69 & 5.8 \\
\hline 298.15 & 2.9303 & 46.03 & 45.66 & 0.80 & 43.30 & 5.9 & 43.44 & 5.6 \\
\hline 303.15 & 2.9298 & 55.08 & 56.62 & -2.8 & 53.72 & 2.5 & 51.75 & 6.0 \\
\hline 308.15 & 2.9288 & 70.85 & 70.23 & 0.88 & 66.43 & 6.2 & 66.56 & 6.1 \\
\hline \multicolumn{9}{|c|}{$x 02=0.402$} \\
\hline 278.15 & 4.0173 & 33.30 & 33.05 & 0.76 & 34.62 & -4.0 & 34.05 & -2.3 \\
\hline 283.15 & 4.0169 & 38.83 & 40.35 & -3.9 & 42.76 & -10 & 39.80 & -2.5 \\
\hline 288.15 & 4.0160 & 49.69 & 49.35 & 0.67 & 52.68 & -6.0 & 50.88 & -2.4 \\
\hline 293.15 & 4.0150 & 62.43 & 60.45 & 3.2 & 64.77 & -3.7 & 63.93 & -2.4 \\
\hline 298.15 & 4.0140 & 74.84 & 74.15 & 0.91 & 79.50 & -6.2 & 76.71 & -2.5 \\
\hline 303.15 & 4.0129 & 88.11 & 91.06 & -3.4 & 97.41 & -11 & 90.51 & -2.7 \\
\hline 308.15 & 4.0109 & 113.1 & 111.9 & 1.1 & 118.9 & -5.1 & 116.2 & -2.7 \\
\hline \multicolumn{9}{|c|}{$x 02=0.5003$} \\
\hline 278.15 & 4.9969 & 60.99 & 60.08 & 1.5 & 58.43 & 4.2 & 60.74 & 0.41 \\
\hline 283.15 & 4.9959 & 70.70 & 72.92 & -3.1 & 71.20 & -0.71 & 70.37 & 0.46 \\
\hline 288.15 & 4.9942 & 87.82 & 88.52 & -0.79 & 86.60 & 1.4 & 87.42 & 0.46 \\
\hline 293.15 & 4.9919 & 110.7 & 107.4 & 3.0 & 105.2 & 5.0 & 110.2 & 0.47 \\
\hline 298.15 & 4.9898 & 131.4 & 130.4 & 0.80 & 127.7 & 2.8 & 130.8 & 0.50 \\
\hline 303.15 & 4.9876 & 154.4 & 158.2 & -2.5 & 154.9 & -0.40 & 153.5 & 0.55 \\
\hline 308.15 & 4.9837 & 193.3 & 191.8 & 0.77 & 187.3 & 3.1 & 192.2 & 0.56 \\
\hline \multicolumn{9}{|c|}{$x 02=0.5993$} \\
\hline 278.15 & 5.9826 & 87.17 & 85.57 & 1.8 & 84.97 & 2.5 & 87.21 & -0.043 \\
\hline 283.15 & 5.9812 & 98.08 & 102.4 & -4.4 & 102.6 & -4.6 & 98.13 & -0.051 \\
\hline 288.15 & 5.9784 & 121.9 & 122.9 & -0.87 & 123.8 & -1.6 & 121.9 & -0.051 \\
\hline 293.15 & 5.9745 & 154.5 & 148.0 & 4.2 & 149.1 & 3.5 & 154.6 & -0.052 \\
\hline 298.15 & 5.9714 & 180.5 & 178.6 & 1.1 & 180.0 & 0.28 & 180.6 & -0.058 \\
\hline 303.15 & 5.9680 & 208.3 & 216.0 & -3.7 & 217.3 & -4.4 & 208.4 & -0.065 \\
\hline 308.15 & 5.9613 & 264.8 & 261.8 & 1.2 & 261.3 & 1.3 & 265.0 & -0.064 \\
\hline
\end{tabular}

${ }^{a}$ The reported results were the average values of three determinations;

${ }^{b}$ The standard uncertainty of temperature is $u(T)=0.03 \mathrm{~K}$;

${ }^{c}$ The relative standard uncertainties are $u_{\mathrm{r}}\left(x_{1}\right)=0.05, u_{\mathrm{r}}\left(x_{2}\right)=0.05, u_{\mathrm{r}}(x 02)=0.02$; the standard uncertainty of the pressure is $u(p)=10 \mathrm{kPa}$;

${ }^{d} x_{2}$ is the mole fraction of methanol in the final saturated ternary mixture; $x 02$ is the initial mole fraction of methanol in methanol + water mixture;

${ }^{e} \mathrm{RD}$ is the relative deviation;

${ }^{f}$ The residual solid phase of cefoxitin acid in the solubility experiments was cefoxitin acid monohydrate. 


\section{Table 4}

Parameters of the modified Apelblat equation for the solubility of cefoxitin acid in methanol, acetonitrile, ethanol, isopropanol, n-propanol and ethyl acetate. ${ }^{a, b, c}$

\begin{tabular}{cccccc}
\hline Solvent & $A^{a}$ & $B^{a}$ & $C^{a}$ & $\mathrm{R}^{2 b}$ & $10^{2} \mathrm{ARD}^{c}$ \\
\hline Methanol & -65.070 & 232.67 & 10.490 & 0.9983 & 0.81 \\
Acetonitrile & -192.55 & 6881.8 & 28.449 & 0.9962 & 0.74 \\
Ethanol & 180.16 & -10857 & -26.467 & 0.9934 & 1.18 \\
Isopropanol & 506.80 & -27056 & -74.526 & 0.9885 & 4.26 \\
n-propanol & -209.64 & 6433.7 & 31.635 & 0.9930 & 1.68 \\
Ethyl acetate & -100.31 & 3249.9 & 14.259 & 0.9935 & 0.55 \\
\hline
\end{tabular}

${ }^{a} A, B, C$ are the parameters of the modified Apelblat equation;

${ }^{b} \mathrm{R}^{2}$ is the coefficient of determination;

${ }^{c} \mathrm{ARD}$ is the average relative deviation.

\section{Table 5}

Parameters of the NRTL model for the solubility of cefoxitin acid in methanol, acetonitrile, ethanol, isopropanol, n-propanol and ethyl acetate. ${ }^{a, b}$

\begin{tabular}{ccccc}
\hline Solvent & $\Delta g_{12}{ }^{a}$ & $\Delta g_{21}{ }^{a}$ & $\alpha_{12}{ }^{a}$ & $10^{2} \mathrm{ARD}^{b}$ \\
\hline Methanol & -51993 & 81734 & 0.025 & 2.62 \\
Acetonitrile & -56757 & 100905 & 0.025 & 1.81 \\
Ethanol & 126982 & -35939 & 0.025 & 2.13 \\
Isopropanol & -43934 & 71676 & 0.025 & 7.40 \\
n-propanol & -52196 & 89674 & 0.025 & 2.30 \\
Ethyl acetate & -58715 & 108382 & 0.025 & 2.70 \\
\hline
\end{tabular}

${ }^{a} \Delta g_{12}, \Delta g_{21}$ and $\alpha_{12}$ are the parameters of the NRTL equation;

${ }^{b} \mathrm{ARD}$ is the average relative deviation.

\section{Table 6}

Parameters of the modified Apelblat equation for the solubility of cefoxitin acid in water and methanol + water mixtures at different initial mole fractions of methanol $\left.\left(\begin{array}{ll}x & 2\end{array}\right)\right)^{a, b}$

\begin{tabular}{ccccc}
\hline$x 02$ & $A^{a}$ & $B^{a}$ & $C^{a}$ & $10^{2} \mathrm{ARD}^{b}$ \\
\hline Water & -295.950 & 8849.94 & 45.0445 & 1.98 \\
\hline
\end{tabular}




\begin{tabular}{lllll}
\hline 0.1013 & -31.9812 & -2445.94 & 5.50571 & 0.89 \\
0.2011 & 10.6099 & -4138.98 & -0.86920 & 1.92 \\
0.2933 & -172.833 & 4098.29 & 26.5719 & 1.65 \\
0.4020 & -185.574 & 4842.02 & 28.4554 & 1.98 \\
0.5003 & -142.868 & 3139.01 & 22.0614 & 1.78 \\
0.5993 & -199.374 & 5732.69 & 30.5074 & 2.47 \\
\hline
\end{tabular}

${ }^{a} A, B, C$ are the parameters of the modified Apelblat equation;

${ }^{b} \mathrm{ARD}$ is the average relative deviation.

\section{Table 7}

Parameters of the NRLT model for the solubility of cefoxitin acid in water and methanol + water mixtures. ${ }^{a, b}$

\begin{tabular}{cccc}
\hline Parameters & Values & Parameters & Values \\
\hline$\Delta g_{12}{ }^{a}$ & -36744 & $\alpha_{12}{ }^{a}$ & 0.025 \\
$\Delta g_{13}{ }^{a}$ & 106763 & $\alpha_{13}{ }^{a}$ & 0.025 \\
$\Delta g_{23}{ }^{a}$ & -25082 & $\alpha_{23}{ }^{a}$ & 0.025 \\
$\Delta g_{21}{ }^{a}$ & 59153 & \multicolumn{2}{c}{$10^{2} \mathrm{ARD}^{b}=7.18$} \\
$\Delta g_{31}{ }^{a}$ & -31229 & & \\
$\Delta g_{32}{ }^{a}$ & -24978 & & \\
\hline
\end{tabular}

${ }^{a} \Delta g_{12}, \Delta g_{13}, \Delta g_{23}, \Delta g_{21}, \Delta g_{31}, \Delta g_{32}, \alpha_{12}, \alpha_{13}$ and $\alpha_{23}$ are the parameters of the NRTL equation;

${ }^{b} \mathrm{ARD}$ is the average relative deviation.

\section{Table 8}

Parameters of the CNIBS/R-K model for the solubility of cefoxitin acid in water and (methanol + water) mixtures. ${ }^{a, b}$

\begin{tabular}{ccccccc}
\hline$T / \mathrm{K}$ & $B_{0}{ }^{a}$ & $B_{1}{ }^{a}$ & $B_{2}{ }^{a}$ & $B_{3}{ }^{a}$ & $B_{4}{ }^{a}$ & $10^{2} \mathrm{ARD}^{b}$ \\
\hline 278.15 & -10.8749 & 15.8208 & -54.1776 & 116.4181 & -87.2201 & 6.11 \\
283.15 & -10.6559 & 16.1752 & -58.1972 & 126.4758 & -95.2406 & 6.04 \\
288.15 & -10.2895 & 13.8061 & -44.6419 & 97.0002 & -73.9492 & 4.92 \\
293.15 & -10.0941 & 15.0459 & -51.5239 & 110.4736 & -82.7065 & 5.47 \\
298.15 & -9.7684 & 14.1842 & -49.5196 & 108.3484 & -82.0530 & 5.14 \\
303.15 & -9.6049 & 14.7845 & -53.3034 & 116.4815 & -88.0379 & 5.38 \\
308.15 & -10.8749 & 15.8208 & -54.1776 & 116.4181 & -87.2201 & 6.11 \\
\hline
\end{tabular}

${ }^{a} B_{0}, B_{1}, B_{2}, B_{3}, B_{4}$ are the parameters of the CNIBS/R-K equation;

${ }^{b} \mathrm{ARD}$ is the average relative deviation.

\section{Table 9}


Molar enthalpy $\left(\Delta_{\text {sol }} H\right)$ of the cefoxitin acid dissolution process in methanol, acetonitrile, ethanol, isopropanol, n-propanol and ethyl acetate. ${ }^{a}$

\begin{tabular}{|c|c|c|c|}
\hline$T / \mathrm{K}$ & $\begin{array}{c}\Delta_{\mathrm{sol}} H \\
/\left(\mathrm{kJ} \cdot \mathrm{mol}^{-1}\right)\end{array}$ & $T / \mathrm{K}$ & $\begin{array}{c}\Delta_{\mathrm{sol}} H \\
/\left(\mathrm{kJ} \cdot \mathrm{mol}^{-1}\right)\end{array}$ \\
\hline \multicolumn{2}{|c|}{ Methanol } & \multicolumn{2}{|c|}{ Acetonitrile } \\
\hline 278.15 & 18.81 & 278.15 & 8.70 \\
\hline 283.15 & 18.72 & 283.15 & 9.70 \\
\hline 288.15 & 18.50 & 288.15 & 10.71 \\
\hline 293.15 & 18.07 & 293.15 & 11.70 \\
\hline 298.15 & 17.48 & 298.15 & 12.70 \\
\hline 303.15 & 16.83 & 303.15 & 13.67 \\
\hline 308.15 & 16.13 & 308.15 & 14.61 \\
\hline \multicolumn{2}{|c|}{ Ethanol } & \multicolumn{2}{|c|}{ Isopropanol } \\
\hline 278.15 & 24.28 & 278.15 & 21.60 \\
\hline 283.15 & 24.64 & 283.15 & 22.22 \\
\hline 288.15 & 25.02 & 288.15 & 22.83 \\
\hline 293.15 & 25.40 & 293.15 & 23.43 \\
\hline 298.15 & 25.76 & 298.15 & 24.04 \\
\hline 303.15 & 26.15 & 303.15 & 24.63 \\
\hline 308.15 & 26.52 & 308.15 & 25.21 \\
\hline \multicolumn{2}{|c|}{ n-propanol } & \multicolumn{2}{|c|}{ Ethyl acetate } \\
\hline 278.15 & 19.56 & 278.15 & 23.48 \\
\hline 283.15 & 20.16 & 283.15 & 23.80 \\
\hline 288.15 & 20.77 & 288.15 & 24.11 \\
\hline 293.15 & 21.35 & 293.15 & 24.44 \\
\hline 298.15 & 21.93 & 298.15 & 24.75 \\
\hline 303.15 & 22.45 & 303.15 & 25.07 \\
\hline 308.15 & 23.01 & 308.15 & 25.36 \\
\hline
\end{tabular}

${ }^{a}$ Combined expanded uncertainties $U$ are $U_{\mathrm{c}}\left(\Delta_{\mathrm{sol}} H\right)=0.040 \Delta_{\mathrm{sol}} H$ ( 0.95 level of confidence).

\section{Table 10}

Molar enthalpy $\left(\Delta_{\mathrm{sol}} H\right)$ of the cefoxitin acid dissolution process in water and methanol + water mixtures at different initial mole fractions of methanol $(x 02){ }^{a}$

\begin{tabular}{|c|c|c|c|}
\hline$T / \mathrm{K}$ & $\begin{array}{c}\Delta_{\mathrm{sol}} H \\
/\left(\mathrm{kJ} \cdot \mathrm{mol}^{-1}\right)\end{array}$ & $T / \mathrm{K}$ & $\begin{array}{c}\Delta_{\mathrm{sol}} H \\
\left(\mathrm{~kJ} \cdot \mathrm{mol}^{-1}\right)\end{array}$ \\
\hline \multicolumn{2}{|c|}{ Water } & \multicolumn{2}{|c|}{$x 02=0.1013$} \\
\hline 278.15 & 30.60 & 278.15 & 33.07 \\
\hline 283.15 & 32.48 & 283.15 & 33.29 \\
\hline 288.15 & 34.36 & 288.15 & 33.52 \\
\hline
\end{tabular}




\begin{tabular}{|c|c|c|c|}
\hline 293.15 & 36.24 & 293.15 & 33.75 \\
\hline 298.15 & 38.12 & 298.15 & 33.98 \\
\hline 303.15 & 40.00 & 303.15 & 34.21 \\
\hline 308.15 & 41.90 & 308.15 & 34.43 \\
\hline \multicolumn{2}{|c|}{$x 02=0.2011$} & \multicolumn{2}{|c|}{$x 02=0.2933$} \\
\hline 278.15 & 32.40 & 278.15 & 27.37 \\
\hline 283.15 & 32.36 & 283.15 & 28.47 \\
\hline 288.15 & 32.32 & 288.15 & 29.58 \\
\hline 293.15 & 32.29 & 293.15 & 30.68 \\
\hline 298.15 & 32.25 & 298.15 & 31.78 \\
\hline 303.15 & 32.21 & 303.15 & 32.88 \\
\hline 308.15 & 32.17 & 308.15 & 33.98 \\
\hline \multicolumn{2}{|c|}{$x 02=0.402$} & \multicolumn{2}{|c|}{$x 02=0.5003$} \\
\hline 278.15 & 25.54 & 278.15 & 24.90 \\
\hline 283.15 & 26.72 & 283.15 & 25.82 \\
\hline 288.15 & 27.90 & 288.15 & 26.73 \\
\hline 293.15 & 29.08 & 293.15 & 27.64 \\
\hline 298.15 & 30.26 & 298.15 & 28.55 \\
\hline 303.15 & 31.43 & 303.15 & 29.46 \\
\hline 308.15 & 32.61 & 308.15 & 30.36 \\
\hline \multicolumn{2}{|c|}{$x 02=0.5993$} & & \\
\hline 278.15 & 22.87 & & \\
\hline 283.15 & 24.13 & & \\
\hline 288.15 & 25.39 & & \\
\hline 293.15 & 26.65 & & \\
\hline 298.15 & 27.91 & & \\
\hline 303.15 & 29.17 & & \\
\hline 308.15 & 30.41 & & \\
\hline
\end{tabular}

${ }^{a}$ Combined expanded uncertainties $U$ are $U_{\mathrm{c}}\left(\Delta_{\mathrm{sol}} H\right)=0.040 \Delta_{\mathrm{sol}} H$ ( 0.95 level of confidence). 